\title{
Maus tratos nos idosos Abordagem nos Cuidados de Saúde Primários
}

\author{
Isabel Costa, ${ }^{*}$ Ângela Pimenta, ${ }^{* *}$ Diana Brigas, $* * *$ Luísa Santos, $* * * *$ Sofia Almeida, ${ }^{* * * * *}$
}

\section{RESUMO}

Objectivo: O progresso da Medicina permitiu o aumento da esperança e qualidade de vida. Simultaneamente, as alterações nos contextos social e familiar modificaram o estatuto social do idoso. Estudos recentes revelam que a violência contra idosos está a aumentar em Portugal (dados da Associação Portuguesa de Apoio à Vítima indicam aumento de 20,4\% no total de idosos vítimas de crime, de 2006 para 2007). Para muitos idosos, a consulta no Médico de Família (MF) é a única oportunidade para detectar maus-tratos.

Este trabalho tem como objectivo rever estratégias de prevenção, identificação e intervenção em situações de maus-tratos nos idosos, em cuidados de saúde primários (CSP).

Fontes de dados: Medline e sites de medicina baseada na evidência.

Métodos de revisão: Efectuou-se uma pesquisa de artigos de revisão e normas de orientação clínica na Medline e sites de medicina baseada na evidência (National Clearinghouse, The Cochrane Library, Bandolier), publicados entre 1998 e 2008 , em português, inglês e espanhol, com as palavras-chave (termos $\mathrm{MESH}$ ): «elder abuse» e «primary care».

Resultados: Foram encontrados 13 artigos, dos quais foram seleccionados 7: 3 revisões sistemáticas, 3 ensaios clínicos aleatorizados e controlados e 1 norma de orientação clínica. Há vários tipos de maus-tratos: físicos; psicológicos; negligência; abuso económico e auto-negligência. A prevenção primária passa pela identificação dos factores de risco (FR), no idoso (comorbilidades, deterioração cognitiva, isolamento social...) e no cuidador (sobrecarga física e emocional, toxicodependência...). A prevenção secundária consiste na identificação dos maus-tratos (colocação de perguntas-chave, detecção de sinais de maus-tratos e avaliação da relação idoso-cuidador) e na instituição de medidas que evitem a sua perpetuação. O MF, em colaboração com Enfermeiros, Assistentes Sociais e Psicólogos, poderá intervir de diversas formas: aconselhar frequência de centro-de-dia, apoio domiciliário, recurso a Unidades de Cuidados Continuados, etc.

Conclusões: Os MF estão em condição privilegiada para reconhecer situações de maus tratos e intervir preventivamente, identificando idosos que necessitam de protecção ou famílias incapazes de prestar cuidados. Dada a falta de normas de orientação e de legislação específica, considera-se importante a realização de protocolos que poderiam potenciar a capacidade de intervenção nesta problemática, nos CSP.

Palavras-chave (termos MESH): Elder Abuse; Primary Care.

\section{INTRODUÇÃO}

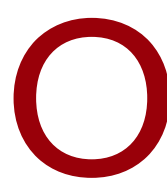

progresso da Medicina tem permitido um aumento da esperança e da qualidade de vida, nunca vividos antes. Para além disso, nas últimas décadas, têm-se verificado alterações na estrutura social e no contexto familiar que conduziram a uma modificação do estatuto social do idoso. Para muitos, este deixou de ser reconhecido como o «sábio», uma fonte de experiência e conheci-

\footnotetext{
*Interna $2^{\circ}$ ano de MGF, C.S. Soares dos Reis - USF Camélias

**Interna $2^{\circ}$ ano de MGF, C.S. Castêlo da Maia - USF Viver Mais

***Interna $2^{\circ}$ ano de MGF, C.S. Soares dos Reis - Unidade de Oliveira do Douro

****Interna $2^{\circ}$ ano de MGF, USF Sete Caminhos - Gondomar

*****Interna $2^{\circ}$ ano de MGF, C.S. Soares dos Reis - USF Nova Salus
}

mento, passando a ser encarado como um «fardo» que é necessário cuidar. Também a falta de preparação de familiares/cuidadores e instituições para lidar com as questões sociais e psíquicas próprias do envelhecimento têm conduzido ao aumento de situações de maus-tratos nos idosos.

Estudos recentes revelam que o fenómeno da violência contra as pessoas idosas está a aumentar em Portugal. Segundo dados publicados pela Associação Portuguesa de Apoio à Vítima (APAV), do ano de 2006 a 2007 verificou-se um aumento de 20,4 porcento do total de pessoas idosas vítimas de crime: de 545 vítimas registadas em 2006, passou-se a observar 656 vítimas em 2007. Segundo a mesma fonte, entre 2000 e 2007 a APAV 
recebeu 3.459 pessoas idosas vítimas de crime. ${ }^{1}$

Para muitos idosos, a consulta no Médico de Família pode ser a única oportunidade para a detecção dos maus-tratos. Infelizmente, muitos médicos atribuem as consequências do abuso (lesões corporais, depressão, mau controlo de doenças crónicas, incapacidade funcional...) ao próprio envelhecimento ou a doenças subjacentes.

Assim, considerando que quanto maior o conhecimento, esclarecimento e a discussão das questões relacionadas com a violência, melhor será a prevenção, a identificação e a actuação nas suas várias manifestações, julgamos pertinente a revisão sobre este tema.

O objectivo deste trabalho é rever estratégias de prevenção, identificação e intervenção em situações de maus-tratos nos idosos, em cuidados de saúde primários (CSP).

\section{MÉTODOS}

Foi efectuada uma pesquisa de artigos de revisão e normas de orientação clínica na Medline e sites de Medicina Baseada na Evidência, publicados entre 1998 e 2008, nas linguas portuguesa, inglesa e espanhola. As palavras-chave utilizadas foram: «elder abuse»e «primary care».

De um total de treze artigos, seis foram excluídos por não se relacionarem com o tema, sendo seleccionados sete (três artigos de revisão, três estudos originais e uma guideline).

\section{RESULTADOS}

\section{Enquadramento}

Pessoas com idade igual ou superior a 65 anos são, em geral, designadas como idosas, embora diversas organizações, nomeadamente a Organização Mundial de Saúde (OMS), utilizem o limite de 60 anos para o fazer.

De acordo com o Artigo $72 .^{\circ}$ da Constituição da República Portuguesa:

1. As pessoas idosas têm direito à segurança económica e a condições de habitação e convívio familiar e comunitário que respeitem a sua autonomia pessoal e evitem e superem o isolamento ou a marginalização social.

2. A política de terceira idade engloba medidas de carácter económico, social e cultural, tendentes a proporcionar às pessoas idosas oportunidades de reali- zação pessoal, através de uma participação activa na vida da comunidade.

O progressivo envelhecimento populacional tem sido acompanhado de mudanças sociais: famílias cada vez mais reduzidas; maior número de mulheres (tradicionalmente responsáveis pelos cuidados aos idosos) inseridas no mercado de trabalho; mudanças culturais com maior tendência à desvinculação; diminuição da tolerância; aumento da descriminação e da institucionalização dos idosos. Todo este contexto torna os maus-tratos aos idosos um problema actual. ${ }^{2}$

Segundo o artigo $152^{\circ}$-A do Código Penal (Maus tratos), «quem, tendo ao seu cuidado, à sua guarda, sob a responsabilidade da sua direcção ou educação, ou a trabalhar ao seu serviço, pessoa menor ou particularmente indefesa, em razão de idade, deficiência, doença ou gravidez, e: a) lhe infligir (...) maus tratos físicos ou psíquicos (...) ou a tratar cruelmente; b) a empregar em actividades perigosas, desumanas ou proibidas; ou c) a sobrecarregar com trabalhos excessivos; é punido com pena de prisão de 1 a 5 anos...». De salientar que, nos casos intra-familiares não se deve falar em maustratos mas antes em violência doméstica, de acordo com as alterações introduzidas pelo novo código penal (artigo $152^{\circ}$ do Código Penal).

Os maus-tratos na terceira idade são ainda definidos pela OMS como um acto único ou repetido, ou ainda, ausência de acção apropriada que cause dano, sofrimento ou angústia e que ocorram dentro de um relacionamento de confiança. ${ }^{3}$

São formas de maus-tratos: ${ }^{4,5}$

- Maus-tratos físicos: qualquer forma de agressão física (espancamentos, golpes, queimaduras, fracturas, administração abusiva de fármacos ou tóxicos, relações sexuais forçadas);

- Maus-tratos psicológicos ou emocionais: condutas que causam dano psicológico como manipulação, ameaças, humilhações, chantagem afectiva, desprezo ou privação do poder de decisão, negação do afecto, isolamento e marginalização;

- Negligência: não satisfazer as necessidades básicas (negação de alimentos, cuidados higiénicos, habitação, segurança e cuidados médicos);

- Abuso económico: impedir o uso e controlo do próprio dinheiro, exploração financeira e chantagem económica; 
- Auto-negligência: incapacidade de um indivíduo desempenhar tarefas de auto-cuidado.

\section{Papel do Médico de Família}

Os Médicos de Família, através do seu contacto continuado com famílias e idosos, estão em posição privilegiada para não só identificar as situações de maus tratos, como para intervir preventivamente reconhecendo as situações de risco, identificando os idosos que necessitam de protecção ou as famílias em que a capacidade de dar apoio / cuidados está comprometida.

Em Portugal cada profissional tem a obrigação legal de denunciar as situações de maus-tratos (alínea b do artigo $242^{\circ}$ do Código do Processo Penal - «a denúncia é obrigatória, ainda que os agentes do crime não sejam conhecidos...para os funcionários... quanto a crimes de que tomaram conhecimento no exercício das suas funções e por causa delas»). Os Médicos têm também obrigação deontológica de o fazer:artigo $44^{\circ}$ do Código Deontológico Médico (Protecção de diminuídos e incapazes): «Sempre que o Médico chamado a tratar uma criança, um idoso, um deficiente ou um incapaz, verifique que estes são vitimas de sevícias, maus tratos ou malévolas provações, deve tomar providências adequadas para os proteger, nomeadamente alertando as autoridades policiais ou as instâncias sociais competentes.»

\section{Prevenção primária}

A prevenção primária de maus-tratos no idoso passa pela identificação e tentativa de modificação dos factores de risco.

Existem alguns factores de risco que contribuem para que o idoso seja vitima de maus-tratos, como:2,4,5

- Idade avançada;

- Existência de comorbilidades;

- Limitação funcional;

- Deterioração cognitiva, alterações psicológicas e do comportamento;

- Dependência física e emocional do idoso relativamente ao cuidador;

- Escassos recursos económicos;

- Reduzido nível educacional;

- Condições de salubridade precárias;

- Isolamento social;

- Antecedentes pessoais ou familiares de maus-tratos.
Para além destes, existem ainda factores de risco relacionados com o cuidador: ${ }^{4,5}$

- Sobrecarga física e emocional;

- Doença psiquiátrica (depressão, burnout, perturbação de personalidade);

- Dependência de substâncias (álcool ou outras drogas);

- Antecedentes pessoais ou familiares de maus-tratos;

- Baixos recursos económicos, dependência económica do cuidador em relação ao idoso.

Outras situações podem ainda ser consideradas de risco: ${ }^{2,45}$

- Existência de cuidador formal (contratado), figura externa, cada vez mais frequente nas famílias, dadas as alterações culturais e sociais que dificultam aos familiares a prestação de cuidados aos seus idosos;

- Relação conflituosa entre o idoso e o cuidador;

- Falta de apoio familiar, social e financeiro.

\section{Prevenção secundária}

A prevenção secundária consiste na detecção precoce dos maus-tratos (pela identificação dos sinais de alerta) e na instituição de medidas que evitem a sua perpetuação.

A U.S. Preventive Services Task Force (USPSTF) e a Canadian Task Force on Preventive Health Care (CTF) concluíram que não há evidência científica suficiente para recomendar o uso de instrumentos de rastreio (questionários) na detecção de maus-tratos no idoso. No entanto, aconselham os profissionais de saúde a estarem atentos e sugerem que se coloquem perguntas durante a entrevista clínica para identificar precocemente factores de risco e sinais de maus-tratos. ${ }^{5}$

Durante a consulta, dever-se-á entrevistar o idoso sozinho e posteriormente na presença do seu cuidador. Durante a entrevista deve-se criar empatia, confiança e facilitar a expressão de sentimentos do idoso; garantir confidencialidade; utilizar uma linguagem acessível e clara; verificar se há mudança de atitude do idoso quando o cuidador está presente e analisar a relação entre eles. ${ }^{6}$

No Quadro I apresentam-se as perguntas sugeridas pela American Medical Association (AMA) e pela CTF para despiste de maus-tratos. ${ }^{6}$

Os maus-tratos em idosos podem ocorrer sob múltiplas formas, cujas consequências frequentemente se 
QUADRO I. Questionário para Despiste de Maus-Tratos no Idoso

1. Já alguma das pessoas com quem vive o magoou?

2. Sente medo de alguma das pessoas com quem vive?

3. Já foi ameaçado por alguém?

4. Já foi obrigado a assinar algum documento que não compreendesse?

5. Já o impediram de tomar decisões por si próprio?

6. Já o tocaram sem o seu consentimento?

7. Já o obrigaram a agir contra a sua vontade?

8. Já lhe retiraram algo que lhe pertencesse sem a sua autorização?

9. Passa muito tempo sozinho?

Adapt. de Fernández-Alonso MC, Herrero-Velázquez S. Maltrato en el anciano: posibilidades de intervención desde la atención primaria (II). Aten Primaria 2006 Feb; 37 (2): 113-5.

sobrepõem e se confundem com sinais e sintomas de patologias prevalentes nessa faixa etária.

Devem-se explorar cuidadosamente os sinais sugestivos de maus-tratos (Quadro II). ${ }^{6,7}$

Todos os dados relevantes colhidos durante a anamnese e o exame objectivo deverão ser registados detalhadamente no processo clínico do idoso.

\section{Formas de Intervenção}

Após identificação dos factores de risco, o Médico de Família poderá intervir no sentido de os modificar, tanto em prevenção primária como secundária.

É importante fazer-se uma revisão de esquemas terapêuticos, tendo em conta o preço, a simplificação da posologia e a adequação do modo de administração.

Com o apoio do Serviço Social pode aconselhar-se a frequência dum centro-de-dia ou recomendar-se cuidados de apoio domiciliário, na tentativa de contrariar o isolamento social. A avaliação das condições habitacionais e da sua adequação às limitações do idoso é também importante. ${ }^{6}$

Pode-se ainda intervir em relação ao cuidador, identificando e actuando preventivamente perante situações de sobrecarga física e emocional que possam fazer despoletar actos de violência contra o idoso, através das seguintes propostas: ${ }^{6}$

- Partilhar os cuidados do idoso com outros membros da família;

\section{QUADRO II. Sinais de Maus-Tratos no Idoso}

- Hematomas, fracturas, lacerações não explicadas;

- Lesões em vários estados de cura;

- Higiene oral e higiene pessoal precárias;

- Perda de peso, desidratação, desnutrição;

- Apatia, depressão ou agravamento de demência;

- Exames auxiliares de diagnóstico não congruentes com a história relatada;

- Fraca adesão ou resposta à terapêutica instituída;

- Quedas frequentes;

- Recorrência frequente aos serviços de urgência por motivos diversos;

- Demora na procura de cuidados de saúde em situações agudas;

- Explicação não plausível para a lesão;

- Disparidade na explicação dada para as lesões pela vítima e pelo abusador suspeito;

- Recusa do cuidador em deixar o idoso sozinho com o profissional de saúde.

Adapt. de Levine JM. Elder neglect and abuse: a primer for primary care physicians. Geriatrics 2003 Oct; 58 (10): 37-44.

- Garantir períodos de descanso ao cuidador (recurso a Unidades de Cuidados Continuados, a Serviços Sociais...)

- Contactar com as redes sociais comunitárias (grupos de apoio, voluntariado, associações de doentes ou de familiares).

Se o cuidador sofrer de patologia psiquiátrica ou de dependência de substâncias, deve-se garantir o apoio e tratamento do seu problema. Nunca esquecer que o reforço positivo do cuidador é fundamental.

No âmbito da sua intervenção na comunidade, o Médico de Família, juntamente com a restante equipa de Cuidados de Saúde Primários (Enfermeiros, Assistentes Sociais, Psicólogos), poderá desenvolver programas educativos orientados para o respeito e reconhecimento dos idosos. ${ }^{5}$

A denúncia de uma situação de maus-tratos pelo Médico de Família gera, com frequência, um conflito ético-legal com o paciente e com a sua família. Por um lado, existe obrigação legal e deontológica de denunciar uma situação de maus-tratos (artigo $242^{\circ}$ do Código do Processo Penal, artigo 44o do Código Deontoló- 
gico Médico). Por outro lado, a denúncia sem consentimento do idoso ameaça a relação de confiança entre o médico e o paciente, viola o segredo profissional a que o Médico está obrigado (artigo $68^{\circ}$ do Código Deontológico) e ameaça a relação com o cuidador, a maioria das vezes também seu paciente. Daí que sejam necessários prudência, conhecimento do contexto e respeito pela vontade do idoso, quando este conserva as suas capacidades mentais (o idoso muitas vezes não admite a existência de maus-tratos por medo, vergonha, por pensar que é uma situação transitória e irá melhorar ou pelos fortes laços de afectividade em relação ao cuidador).

No entanto, o dever de denúncia/sinalização é inultrapassável desde que o Médico de Família actue na qualidade de funcionário público e tenha tido conhecimento do caso no exercício da sua actividade profissional.

Assim, o Médico de Família deverá em primeiro lugar, e no sentido de que o idoso decida livremente, tentar esclarecê-lo do risco que corre se não pedir ajuda, explicando-lhe a forma de evolução destes casos; dizer-lhe que existem outros casos semelhantes ao seu; esclarecê-lo que não tem culpa no sucedido e que poderá encontrar apoio (equipa de saúde, instituições, associações e redes de apoio, etc.) e ver a situação resolvida. Se assim mesmo não resultar e o idoso, pelos seus meios, não apresentar queixa, o Médico de Família pode sinalizar o caso ao Ministério Público, pedindo o anonimato para não ferir a necessária relação de confiança médico-doente.

Após avaliação do risco e urgência da situação, das causas, da vontade do idoso e das possibilidades de intervenção, o Médico de Família deverá actuar estabelecendo um plano de acção, que poderá passar pelo contacto com o Assistente Social que articulará com os serviços sociais (por exemplo, Segurança Social) e com os serviços especializados (autoridade de saúde, polícia e associações de protecção à vítima), se necessário.

\section{CONCLUSÕES}

O reconhecimento público dos maus-tratos dos idosos como um problema médico e social é relativamente recente. O Médico de Família está numa situação privilegiada não só para diagnosticar precocemente e abordar eficazmente, mas também para prevenir situações de maus-tratos nas famílias de risco.
O objectivo principal deverá ser a prevenção dos maus-tratos quando possível. Contudo, se as actividades preventivas falharem, devem ser encetadas acções efectivas para pôr fim à situação de maus-tratos. Esta intervenção é complexa e deverá sempre ter em conta uma abordagem multidisciplinar.

Dada a falta de normas de orientação e de legislação específica, considera-se importante a realização e aprovação de protocolos de forma a aumentar a capacidade de detecção e intervenção sobre esta problemática nos Cuidados de Saúde Primários.

\section{CONTACTOS ÚTEIS:}

Associação Portuguesa de Apoio à Vitima - 707200077

PSP/GNR

Tribunal/Ministério Público

Instituto de Medicina Legal

Linha Nacional de Emergência Social - 144

Linha do Cidadão Idoso da Provedoria de Justiça - 800203531

\section{REFERÊNCIAS BIBLIOGRÁFICAS}

1. Apav.pt. Disponível em:http://www.apav.pt/portal/pdf/pessoas_idosas_ vitimas_crime_2000-2008.pdf [acedido em 01/08/2009].

2. Coma M, Muñoz J, Postigo P, Ayuso M, Sierra C, Sayrol T. Visión del maltrato al anciano desde atención primária. Gac Sanit 2007 May- Jun; 21 (3): 235-41.

3. WHO/INPEA. Missing voices: views of older persons on elder abuse. Geneva:WHO; 2002.

4. Hirsch $\mathrm{CH}$, Stratton $\mathrm{S}$, Loewy R. The primary care of elder mistreatment. WEST J MED 1999 Jun; 170 (6): 353-8.

5. Fernández-Alonso MC, Herrero-Velázquez S. Maltrato en el anciano: posibilidades de intervención desde la atención primaria (I). Aten Primaria 2006 Ene; 37 (1):56-9.

6. Fernández-Alonso MC, Herrero-Velázquez S. Maltrato en el anciano: posibilidades de intervención desde la atención primaria (II). Aten Primaria 2006 Feb; 37 (2):113-5.

7. Levine JM. Elder neglect and abuse: a primer for primary care physicians. Geriatrics 2003 Oct; 58 (10): 37-44.

8. Rodriguez MA, Wallace SP, Woolf NH, Mangione CM. Mandatory reporting of elder abuse: between a rock and a hard place. Ann Fam Med 2006 Sep-Oct; 4 (5): 403-9.

9. Kennedy RD. Elder abuse and neglect: the experience, knowledge, and attitudes of primary care physicians. Fam Med 2005 Jul-Aug; 37 (7): 481-5.

Os autores declararam não possuir conflitos de interesses

\section{AGRADECIMENTOS}

As autoras agradecem à Prof. ${ }^{a}$ Dr. ${ }^{a}$ Teresa Magalhães, Professora de Medicina Legal da Universidade do Porto e Directora da Delegação do Norte do Instituto Nacional de Medicina Legal, pela ajuda prestada no esclarecimento de dúvidas de ordem legal. 


\section{ENDEREÇO PARA CORRESPONDÊNCIA}

Isabel Costa

Avenida de Francelos, $N^{\circ} 543$, Bloco 2, $1^{\circ}$ Esq.

4405-700 Gulpilhares, Vila Nova de Gaia

E-mail: isabelmscosta@mail.pt

\section{ABSTRACT}

\section{ELDER MISTREATMENT}

Goals: Progress in medicine has enabled the rise of life expectancy and quality. Meanwhile, changes in social and family contexts changed the social status of the elderly. Recent studies show that violence against elderly people is increasing in Portugal (data from the Portuguese Association for Victim Support - APAV, indicating an increase of $20.4 \%$ of the total elderly victims of crime, from 2006 to 2007). For many elderly, the consultation of the Family Physician (FP) is the only opportunity to detect mistreatment.

This article aims to review strategies for the prevention, identification and intervention in cases of elder mistreatment in primary care (PC).

Data Sources: Medline and evidence-based medicine sites.

Review methods: A search was done of review articles and guidelines in Medline and evidence-based medicine sites (National Clearinghouse, The Cochrane Library, Bandolier), published between 1998 and 2008, in Portuguese, English and Spanish, with the keywords: "Elder abuse" and "primary care".

Results: 13 articles were found, 7 of which were selected: 3 systematic reviews, 3 randomized controlled trials and 1 guideline. There are several types of mistreatment: physical, psychological, neglect, economic abuse and self-neglect. Primary prevention is made through identification of risk factors (RF) in the elderly (comorbidities, cognitive deterioration, social isolation,...) and the caregiver (physical and emotional overload, drug addiction,...). Secondary prevention is the identification of mistreatment (asking key questions, detection of signs of mistreatment signs and evaluation of the relationship between elder and care provider) and the implementation of measures to prevent its perpetuation. The FP, with the help of nurses, social workers and psychologists, may intervene in several ways: advising day care center, home support, Continuous Care Units, etc.

Conclusions: The FP's have the privileged opportunity to recognize elder abuse and to act preventively, identifying elderly in need of protection or families unable to care. Because of the lack of guidelines and specific legislation, it is important to develop protocols that could enhance the ability to deal with this issue, in PC.

Keywords: Elder Abuse; Primary Care. 